Revista Mexicana de Astronomía y Astrofísica, 56, 193-199 (2020)

(C) 2020: Instituto de Astronomía, Universidad Nacional Autónoma de México

https://doi.org/10.22201/ia.01851101p.2020.56.02.02

\title{
A SEARCH FOR PULSATION IN TWENTY-ONE WHITE DWARFS
}

\author{
E. Paunzen ${ }^{1}$, G. Handler ${ }^{2}$, J. Janík ${ }^{1}$, Z. Zemanová ${ }^{3}$, M. Rode-Paunzen ${ }^{4}$, M. S. O’Brien ${ }^{5}$, T. K. Watson ${ }^{6}$, and M. Dróżdż
}

Received February 5 2020; accepted April 242020

\begin{abstract}
Well-defined astrophysical constraints of white dwarfs (WDs), such as on the presence or absence of pulsational variability, are very much needed to refine and develop current models. Because these stars are rather faint and variability periods are mostly below one hour, only a very limited amount of space-based data is currently available for these objects. We present about 68 hours of high-quality ground-based photometric time-series data for twenty-one WDs acquired at five different observatories. No new pulsators were detected but the derived upper limits of variability, which are typically on the order of only a few mmags, provide important input for pulsation models.
\end{abstract}

\section{RESUMEN}

Es muy necesario contar con cotas astrofísicas bien definidas para los parámetros de las enanas blancas (WD) con el objeto de mejorar los modelos actuales. Dado que estas estrellas son débiles y tienen períodos de variabilidad de menos de una hora, actualmente sólo contamos con datos muy limitados basados en observaciones espaciales. Presentamos 68 horas de datos fotométricos de alta calidad obtenidos en cinco obsevatorios terrestres para 21 enanas blancas. No se encontraron pulsadores nuevos, pero los límites superiores para la variabilidad que determinamos, del orden de unas cuantas mmags, son importantes para la construcción de modelos de pulsación.

Key Words: stars: variables: general — white dwarfs

\section{INTRODUCTION}

White dwarfs (WDs) are the last stage of evolution for most stars. Probing their internal structures provides us with important insights on the complex evolutionary paths that led up to this stage. Furthermore, evolution is still ongoing in the WD stage, where it is primarily dominated by cooling (e.g. Salaris et al. 2013). WD interiors are characterized by stratified layers of different masses which mix and settle at certain temperatures. Therefore, as a WD cools down, its surface composition changes (e.g. Tremblay et al. 2013). Consequently, in the different phases (or temperature ranges) of WD evolution, one encounters WDs with H-dominated atmospheres (DAs) and non-DAs (He-atmosphere DOs and DBs).

On its evolutionary path, a WD crosses several instability strips, which are determined by atmospheric composition and total mass. The resulting pulsational vari-

\footnotetext{
${ }^{1}$ Department of Theoretical Physics and Astrophysics, Masaryk University, Czech Republic.

${ }^{2}$ Nicolaus Copernicus Astronomical Center, Poland.

${ }^{3}$ High school, Uherské Hradiště, Czech Republic.

${ }^{4}$ Nicolaus Copernicus Astronomical Center, Poland.

${ }^{5}$ Space Telescope Science Institute, Baltimore, USA.

${ }^{6}$ Information Technology, Southwestern University, USA.

${ }^{7}$ Mt. Suhora Observatory, Poland.
}

ability opens up possibilities to probe the internal structure; for instance, Romero et al. (2014) demonstrated that nonradial gravity mode (g-mode) pulsations allow the study of the stellar interior via the technique of asteroseismology.

Asteroseismology of WDs gained momentum with the work of Winget et al. (1994), who derived precise stellar parameters for the DBV GD 358 from more than 180 significant peaks in its frequency spectrum. The corresponding group of variable stars was later designated V777 Her objects, which pulsate in g-modes with periods between 100 and $1200 \mathrm{~s}$ (Beauchamp et al. 1999). Their cooler cousins, the ZZ Ceti stars, consist of pulsating H-rich DAV WDs and exhibit variability with periods between 70 and 2000 s (Romero et al. 2019). During the last two decades, ground-based and satellite observations of WDs have significantly widened our knowledge in this field of study (Kepler et al. 2005; Hermes et al. 2014) by discovering and analysing many pulsating WDs.

However, several important questions about the pulsational behaviour of WDs remain: What exactly defines the borders of the instability strip? Do all WDs situated inside the instability strip pulsate? If not, what are the important astrophysical parameters? 
TABLE 1

TARGET STARS AND THEIR ASTROPHYSICAL PARAMETERS FROM THE LITERATURE

\begin{tabular}{cccccc}
\hline WD & $\begin{array}{c}T_{\text {eff }} \\
{[\mathrm{K}]}\end{array}$ & $\begin{array}{c}\log g \\
{[\mathrm{dex}]}\end{array}$ & $\log \mathrm{H} / \mathrm{He}$ & $\log \tau$ & Ref \\
\hline $0002+729$ & 14410 & 8.27 & -5.95 & 8.52 & 4 \\
$0017+136$ & 18130 & 8.08 & -4.63 & 8.07 & 4 \\
$0038+555$ & 8860 & 8.00 & & 8.96 & 2 \\
$0231+570$ & 13790 & 8.03 & & 8.43 & 2 \\
$0449+252$ & 11520 & 8.00 & & 8.62 & 2 \\
$0454+620$ & 10960 & 8.88 & & 9.30 & 2 \\
$0713+399$ & 65000 & 7.50 & & & 5 \\
$0835+340$ & 22230 & 8.25 & $<-4.63$ & 7.90 & 4 \\
$0921+091$ & 19470 & 8.01 & -4.72 & 7.90 & 4 \\
$0949+094$ & 18694 & 8.09 & -4.52 & & 3 \\
$1052+273$ & 23930 & 8.41 & & 7.96 & 2 \\
$1107+265$ & 15130 & 8.11 & -5.77 & 8.35 & 4 \\
$1149-133$ & 20370 & 8.30 & -3.77 & 8.08 & 4 \\
$1326-037$ & 19950 & 8.03 & $<-4.81$ & 7.87 & 4 \\
$1540+680$ & 22240 & 7.96 & $<-4.43$ & 7.58 & 4 \\
$1612-111$ & 23430 & 7.96 & $<-4.75$ & 7.46 & 4 \\
$1727+560$ & 12500 & 8.00 & -3.70 & & 1 \\
$2147+280$ & 12940 & 8.86 & -5.74 & 9.08 & 4 \\
$2234+064$ & 23770 & 8.07 & $<-4.72$ & 7.56 & 4 \\
$2246+120$ & 26840 & 7.92 & $<-4.30$ & 7.13 & 4 \\
$2250+746$ & 16560 & 8.15 & $<-6.18$ & 8.26 & 4 \\
\hline
\end{tabular}

1. Wegner \& Koester (1985), 2. Limoges et al. (2015), 3. Koester \& Kepler (2015), 4. Rolland et al. (2018), 5. Werner et al. (2018)

In this paper, we present high-quality ground-based time-series of twenty-one WDs, including one hot Herich DO, with $V$ magnitudes between 13.7 and $16.7 \mathrm{mag}$. These objects are too faint for most current automatic ground-based surveys and at the limit of observability even for satellite-based missions such as Kepler (Doyle et al. 2017). Among the first published results from the TESS mission was an investigation of the known pulsating DBV WD 0158-160 (Bell et al. 2019). With a main period of about $640 \mathrm{~s}$ and an amplitude of about $22 \mathrm{mmag}$, this object is quite typical among pulsating WDs. Generally, TESS data are of limited use in this field of study because of the employed observing cadences ( 2 and $30 \mathrm{~min}$ ), the faintness of most WDs, and the expected amplitudes.

\section{TARGET SELECTION, OBSERVATIONS AND REDUCTION}

We selected interesting WDs situated within or close to the heuristically established instability strip (Althaus et al. 2010). Several He-rich and He-weak objects were included to cover different atmospheric characteristics. In addition, our sample covers a wide range of ages. The astrophysical parameters of our target stars from the literature are listed in Table 1.

Photometric observations were performed at the following four sites using the described telescopes and instruments:

- Hvar Observatory, University of Zagreb, $1.0 \mathrm{~m}$, Austrian-Croatian Telescope (ACT), Apogee Alta U47 CCD camera, integration time $15 \mathrm{~s}$ for all targets, no filter,

- Institute of Astronomy of the University of Vienna, $0.8 \mathrm{~m}$, "vienna little telescope" (vlt), SBIG STL$6303 \mathrm{E}$ CCD camera, integration time 20 to $30 \mathrm{~s}$, no filter,

- McDonald Observatory (McD), 2.1m, Argos, integration time 10 to $25 \mathrm{~s}$ for all targets, BG40 filter,

- Mt. Suhora Observatory (MSO), Poland, 0.6m, Zeiss telescope, Apogee Alta U47 CCD, integration time $30 \mathrm{~s}$ for all targets, no filter,

- South African Astronomical Observatory (SAAO), $0.75 \mathrm{~m}$, high-speed CCD photometer, integration time 5 to $10 \mathrm{~s}$, no filter.

Data reduction and differential photometry were performed using three different program packages:

- Image Reduction and Analysis Facility (IRAF, Stetson 1987),

- Munipack (Chrastina \& Hroch 2008),

- Multiple object and multiple frame CCD photometry (MOMF, Kjeldsen \& Frandsen 1992).

For IRAF and Munipack, classical aperture photometry was applied; the MOMF package combines this with point spread function photometry. None of our target fields were very crowded, but the resulting noise level from each program package was tested. Time-series analysis of the differential light curves was conducted applying a standard Fourier technique and the PhaseDispersion Method. All computations were done within the programme package Peranso (Paunzen \& Vanmunster 2016). To account for different seeing conditions, several apertures were used and tested. For each data set, we chose the aperture which yielded the smallest scatter in the differential light curves. For all objects, several comparison stars were available, which were checked individually to exclude variable objects. 
TABLE 2

OBSERVATION LOG AND RESULTS FROM THE TIME-SERIES ANALYSIS

\begin{tabular}{|c|c|c|c|c|c|c|c|c|c|}
\hline WD & Other & $\begin{array}{c}V \\
{[\mathrm{mag}]}\end{array}$ & $\begin{array}{l}\text { HJD(start) } \\
{[2450000+]}\end{array}$ & $\begin{array}{c}\Delta t \\
{[\mathrm{~min}]}\end{array}$ & $N$ & Site & $\begin{array}{c}\mathrm{UL} \\
{[\mathrm{mmag}]}\end{array}$ & $\begin{array}{c}\text { Freq } \\
{[\mathrm{mHz}]}\end{array}$ & $\begin{array}{c}\text { Amp } \\
{[\mathrm{mmag}]}\end{array}$ \\
\hline $0002+729$ & GD 408 & 14.3 & 8806.221126 & 296.6 & 517 & MSO & 1.2 & & \\
\hline $0017+136$ & Feige 4 & 15.4 & 5418.541842 & 71.0 & 246 & ACT & 4.3 & & \\
\hline $0038+555$ & EGGR 245 & 14.1 & 8807.286154 & 108.8 & 206 & MSO & 3.0 & & \\
\hline $0231+570$ & GD 283 & 13.7 & 8823.262619 & 305.6 & 557 & MSO & 0.8 & & \\
\hline \multirow[t]{2}{*}{$0449+252$} & PM J04523+2519 & 14.9 & 8806.437791 & 189.3 & 335 & MSO & 1.7 & & \\
\hline & & & 8824.394303 & 176.1 & 312 & MSO & 1.8 & & \\
\hline $0454+620$ & PM J04586+6209 & 14.5 & 8823.500997 & 304.6 & 551 & MSO & 1.0 & & \\
\hline \multirow[t]{5}{*}{$0713+399$} & HS 0713+3958 & 16.2 & 2262.816274 & 27.0 & 99 & $\mathrm{McD}$ & 3.4 & & \\
\hline & & & 2263.795979 & 99.5 & 198 & $\mathrm{McD}$ & 1.9 & & \\
\hline & & & 2266.794590 & 99.5 & 198 & $\mathrm{McD}$ & 2.4 & & \\
\hline & & & 2267.801534 & 89.5 & 180 & McD & 2.3 & & \\
\hline & & & 2268.811951 & 69.5 & 140 & $\mathrm{McD}$ & 2.1 & & \\
\hline $0835+340$ & CSO 197 & 16.0 & 4560.591748 & 57.0 & 323 & $\mathrm{McD}$ & 3.6 & & \\
\hline \multirow[t]{3}{*}{$0921+091$} & PG 0921+092 & 16.2 & 4555.278322 & 107.7 & 228 & vlt & 6.6 & & \\
\hline & & & 4557.278634 & 89.7 & 190 & vlt & 7.2 & & \\
\hline & & & 4563.582199 & 55.5 & 310 & $\mathrm{McD}$ & 1.7 & & \\
\hline \multirow[t]{3}{*}{ 0949+094 } & PG 0949+094 & 16.0 & 4205.309769 & 163.3 & 300 & vlt & 3.6 & & \\
\hline & & & 4206.336458 & 124.8 & 224 & vlt & 4.4 & & \\
\hline & & & 4562.600301 & 56.2 & 286 & $\mathrm{McD}$ & 1.8 & & \\
\hline $1052+273$ & GD 125 & 14.2 & 8824.524161 & 251.9 & 425 & MSO & 1.4 & & \\
\hline \multirow[t]{2}{*}{$1107+265$} & GD 128 & 15.9 & 4202.345671 & 108.2 & 194 & vlt & 3.7 & & \\
\hline & & & 4203.310475 & 161.0 & 307 & vlt & 3.4 & & \\
\hline $1149-133$ & HE $1149-1320$ & 16.1 & 3792.577824 & 97.2 & 356 & SAAO & 4.6 & & \\
\hline \multirow[t]{2}{*}{$1326-037$} & PG 1326-037 & 15.7 & 3794.604931 & 63.4 & 529 & SAAO & 5.7 & & \\
\hline & & & 3795.595417 & 74.7 & 428 & SAAO & 1.3 & & \\
\hline $1540+680$ & PG $1540+681$ & 16.2 & 5419.310115 & 94.4 & 249 & ACT & 3.5 & & \\
\hline $1612-111$ & GD 198 & 15.5 & 3790.591169 & 75.2 & 423 & SAAO & 2.8 & & \\
\hline $1727+560$ & G $227-5$ & 16.1 & 5417.399217 & 84.5 & 269 & ACT & 1.9 & & \\
\hline $2147+280$ & EGGR 583 & 14.7 & 8824.189224 & 209.3 & 369 & MSO & 3.1 & & \\
\hline $2234+064$ & PG 2234+064 & 16.0 & 5418.463723 & 104.7 & 369 & ACT & 2.9 & & \\
\hline $2246+120$ & V393 Peg & 16.7 & 5417.477938 & 198.6 & 699 & $\mathrm{ACT}$ & & 3.05 & 15.8 \\
\hline $2250+746$ & GD 554 & 16.7 & 5421.460083 & 88.0 & 318 & ACT & 2.6 & & \\
\hline
\end{tabular}

First, the upper limits of variability of the WDs were compared, which yielded the same overall noise level of $\pm 0.2 \mathrm{mmag}$ for all three methods. No trend of the noise level with frequency was detected. Furthermore, we checked the multiperiodic solution for WD 2246+120. Here, usage of the MOMF package resulted in a slightly better detection probability than provided by the other two packages.

The observation log and results from the time-series analysis are shown in Table 2. In total, we analysed 10335 frames spanning 4103 minutes of observations. All images and data are available on request from the first author.

\section{RESULTS}

No new pulsating WDs were identified among our sample of twenty-one WDs; however, we have derived upper limits of variability that are also important input parameters for the test and development of pulsation models.

The results of the time-series analysis are shown in Figures 1 and 2. Defining the upper limit of variability is not straightforward and has often been discussed in the literature (Reegen et al. 2008). In general, the statistical significance of the noise in the Fourier spectrum is underestimated. We here employ a conservative approach and define the upper limit of variability as the upper envelope of the peaks in an amplitude spectrum (Figures 1 


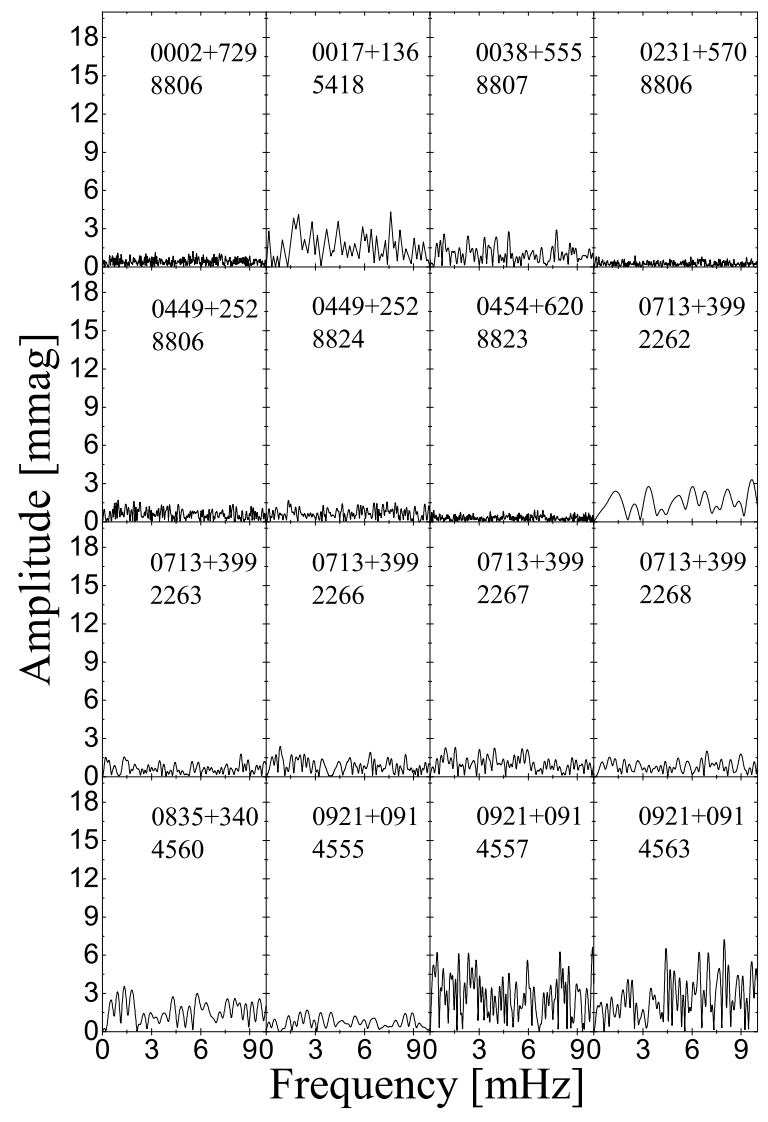

Fig. 1. Fourier spectra of our target stars. Each panel lists the WD number and the JD 2450000+ of the observing night.

and 2). None of the detected amplitudes exceed a significance of $1.3 \sigma$ of the noise level in the corresponding spectrum. Typically, we achieved an upper limit for these targets of a few mmag. In the following, information on the individual stars is provided.

WD 0002+729: This is a carbon-poor and metal-rich WD that does not show any signs of a surrounding dust disk (Farihi et al. 2009). The origin of its peculiar abundance pattern is still not well understood.

WD 0017+136: Limoges et al. (2015) published a parallax-based distance of $33.3 \mathrm{pc}$ from the Sun for this $0.65 M_{\odot}$ object. They concluded that it is one of the seven DBs known to be closer than $40 \mathrm{pc}$. However, the spectroscopy-based distance of $75 \mathrm{pc}$ (Bergeron et al. 2011) does not agree with that, which could be caused by (hitherto undetected) binarity. Bailer-Jones et al. (2018) published a distance of $78.9 \mathrm{pc}$ on the basis of Gaia DR2 (Lindegren et al. 2018). In the latter work, WD 0017+136 is not flagged as a binary. Gaia DR3, which is scheduled for 2021, is going to include a list of binaries, which might finally solve this issue.

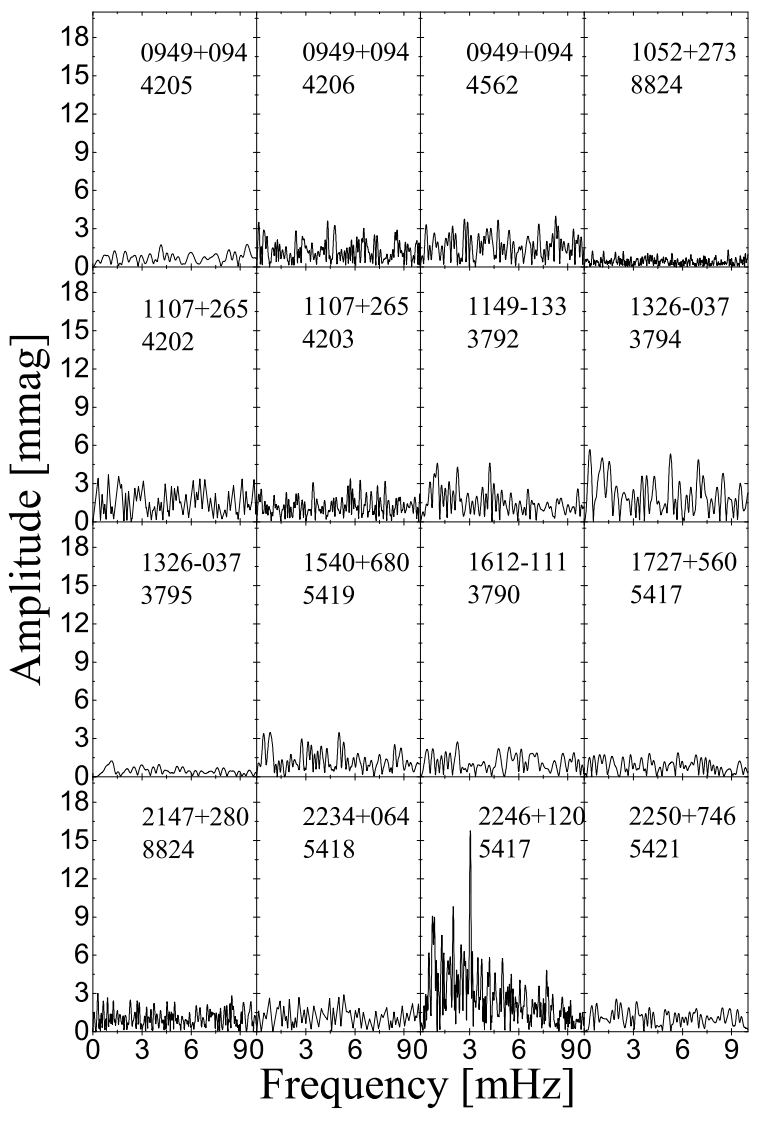

Fig. 2. Fourier spectra of our target stars. Each panel lists the WD number and the JD 2450000+ of the observing night. WD $2246+120$ is a known pulsator immediately noticeable from its rich frequency spectrum.

WD 0038+555: Marinoni et al. (2016) included this object in their photometric short-term variability monitoring survey, which formed a part of the Gaia spectrophotometric standard star calibration. They derived an upper limit of variability of $2 \mathrm{mmag}$ in Johnson $B$ and, within their criteria, accepted WD $0038+555$ as a constant star. Our results are in line with this study.

WD 0231+570: This is one of the UBVRI photoelectric standard stars of Landolt (2013), which were observed within a period of 17 years at the Kitt Peak National and Lowell Observatories. Since no individual errors are given by the authors, an independent check of variability on short timescales is important. We here derive an upper limit of $0.8 \mathrm{mmag}$ for this star.

WD 0449+252 and WD 0454+620: Both stars are included in the paper of Bognár et al. (2018), who searched for new WD pulsators for observations by the TESS space telescope. The authors reported no signs of shortterm variability. 
WD 0713+399: This DO star is remarkable in several respects. Werner et al. (1995) detected unusually strong $\mathrm{He}$ II absorption lines that could not be reproduced by a simple DO model atmosphere. They speculated that the star may belong to a new subgroup of hot He-rich WDs. Later, Dreizler \& Werner (1996) investigated an up-todate list of these WDs and concluded that they represent the non-DA cooling sequence from the hot end down to the DB gap. The observed mean mass value of about $0.6 M_{\odot}$ coincides very well with those of DA and DB WDs.

WD 0835+340: Wagner et al. (1986) were the first to classify this star as a DB3, which was later confirmed by several other studies. Furthermore, Girven et al. (2011) did not find any IR-excess for this object, which implies that no low-mass stellar companion or dusty debris disc is present around it.

WD 0921+091: This star has a very low total hydrogen mass $\left(M_{\mathrm{H}}=6.753 \times 10^{-14} M_{\odot}\right)$ as compared to similar WDs (Voss et al. 2007), which renders it an interesting object for defining constraints on pulsation models. Robinson \& Winget (1983) observed WD 0921+091 photometrically using a high-speed two-star photometer. They presented upper limits of variability (semiamplitude) between 5.6 and $8.0 \mathrm{mmag}$. Comparing their results to ours, it is interesting to note that the $\mathrm{CCD}$ technology delivers results of the same quality as the best classical photoelectric instruments with much smaller telescopes.

WD 0949+094: Castanheira et al. (2006) presented $1.25 \mathrm{~h}$ (193 data points) of photometric observations of this star. However, they did not discover any significant signals in the observed Fourier spectrum (noise of about $6 \mathrm{mmag}$ ). Our limits of variability range between 1.6 and 4.4 mmag (Table 2).

WD 1052+273: Several studies unsuccessfully searched for a dust disk and an IR-excess around this rather bright WD (Wilson et al. 2019).

WD 1107+265: Several studies of this object are available in the literature (Bergeron et al. 2011). The $\log \mathrm{H} / \mathrm{He}$ value is very low (Table 1). Robinson \& Winget (1983) derived upper limits of variability (semiamplitude) between 4.2 and $5.7 \mathrm{mmag}$. Our results are compatible with these values.

WD 1149-133: Detailed analyses from the UV to the IR region are available in the literature for this $\mathrm{He}$ rich WD (Liebert et al. 1986). The results from our single observing run indicate a rather high upper limit of variability ( $4.6 \mathrm{mmag}$ ). The star should be re-observed.

WD 1326-037: This WD is included as constant object in the database of synthetic photometry in the GALEX ultraviolet bands for the stellar sources observed with the International Ultraviolet Explorer (Beitia-Antero \& Gómez de Castro 2016).

WD 1540+680: According to Beauchamp et al. (1999), this object is situated just on the red edge of the instability strip.

WD 1612-111: The search for a low-luminosity companion to this object yielded a null-result (Farihi et al. 2005).

WD 1727+560: The spectrum of this massive DQ WD contains strong emission features of O I and C II (Provencal et al. 2005). Unexpectedly, as compared with carbon, oxygen is overabundant by a factor of about 160 in the chromosphere. In summary, WD $1727+560$ is an outstanding object for which we have not detected any signs of variability.

WD 2147+280: With an age of more than $1 \mathrm{Gyr}$, this star is one of the oldest objects in our sample.

WD 2234+064: This is one of the rare WDs that show a weak Ca II K-line of interstellar origin (Zuckerman et al. 2010).

WD 2246+120: In order to check the reliability of our observational strategy and reduction techniques, we observed this known variable star, which is a pulsating He-atmosphere DB (Handler 2001). It shows variability with periods between 255 and $300 \mathrm{~s}$. The periods and amplitudes derived in the present study are in excellent agreement with the literature values, which lends confidence to our analysis.

WD 2250+746: Robinson \& Winget (1983) derived upper limits of variability (semi-amplitude) between 3.0 and $7.1 \mathrm{mmag}$, which is comparable to our results.

We have compared our derived upper limits of variability (column "UL" in Table 2) with the extensive surveys aimed at the detection of pulsating WDs by Mukadam et al. (2004), Mullally et al. (2005) and Castanheira et al. (2010). These authors also used CCD observations with comparable time bases and integration times. The observations by Mukadam et al. (2004) and Mullally et al. (2005) were done with the same equipment we employed at the McDonald Observatory, whereas Castanheira et al. (2010) used the $4.1 \mathrm{~m}$ SOAR telescope. The limit for non-variability (NOV) was established using a classical Fourier transformation technique defining the highest white-noise peak as the detection threshold. The latter is given in the unit of millimodulation amplitude (mma), which corresponds to a $0.1 \%$ change in intensity. Typically, NOV limits between 2 and $8 \mathrm{mma}$ were reached. This is well within the range of our results. Castanheira et al. (2010) re-observed stars with high NOV limits and detected new variables with amplitudes below the NOV limits among them. Therefore, we caution that there might well be some undetected low-amplitude variables among the WDs of our sample. 


\section{CONCLUSIONS}

The two primary temperature regimes in which isolated cooling WDs are observed to pulsate are $T_{\text {eff }} \approx 12000 \mathrm{~K}$ for the DAVs, and $T_{\text {eff }} \approx 25000 \mathrm{~K}$ for the DBVs. Pulsations are driven in a subsurface partial ionization zone of the dominant surface chemical element, have periods between 70 and $2000 \mathrm{~s}$, and are composed of nonradial gravity modes.

Among the hotter WDs, we find the GW Vir objects with $T_{\text {eff }}$ between $75000 \mathrm{~K}$ and $200000 \mathrm{~K}$ and periods between 300 and $5000 \mathrm{~s}$. Here, the pulsations are driven by ionized carbon and oxygen.

Pulsating WDs provide an important glimpse into the future of the vast majority of stars and their interiors. With asteroseismic tools, it is possible to trace the stellar structure down to the core.

For modeling efforts, it is not only important to analyse the pulsational characteristics of variable WDs but also apparently non-variable objects in the same region of the Hertzsprung-Russell diagram. The search for (dis)similarities between pulsators and non-pulsators can significantly improve the framework of astrophysical parameters in which pulsation occurs.

In this work, we presented high-quality photometric time-series observations of twenty-one WDs from five different sites. To cover interesting regions of the instability strip, our sample encompassed He-rich and Heweak objects. Although we have not detected any new pulsating WD, the derived upper limits of variability are important input parameters for pulsation models.

The authors are grateful to Elisabeth Guggenberger, Patrick Lenz, Stefan Meingast and Monika Lendl for taking part in the observations and to Stefan Hümmerich for helping with the language editing. GH acknowledges support by the Polish NCN grant UMO2015/18/A/ST9/00578.

\section{REFERENCES}

Althaus, L. G., Córsico, A. H., Isern, J., \& García-Berro, E. 2010, A\&ARv, 18, 471

Bailer-Jones, C. A. L., Rybizki, J., Fouesneau, M., Mantelet, G., \& Andrae, R. 2018, AJ, 156, 58

Beauchamp, A., Wesemael, F., Bergeron, P., et al. 1999, ApJ, 516,887

Beitia-Antero, L. \& Gómez de Castro, A. I. 2016, A\&A, 596, A49

Bell, K. J., Córsico, A. H., Bischoff-Kim, A., et al. 2019, A\&A, 632, 42

Bergeron, P., Wesemael, F., Dufour, P., et al. 2011, ApJ, 737, 28

Bognár, Z., Kalup, C., Sódor, Á., Charpinet, S., \& Hermes, J. J. 2018, MNRAS, 478, 2676

Castanheira, B. G., Kepler, S. O., Handler, G., \& Koester, D. 2006, A\&A, 450, 331

Castanheira, B. G., Kepler, S. O., Kleinman, S. J., Nitta, A., \& Fraga, L. 2010, MNRAS, 405, 2561
Chrastina, M. \& Hroch, F. 2008, OEJV, 95, 21

Doyle, T. F., Howell, S. B., Petit, V., \& Lépine, S. 2017, MNRAS, 464, 3464

Dreizler, S. \& Werner, K. 1996, A\&A, 314, 217

Farihi, J., Becklin, E. E., \& Zuckerman, B. 2005, ApJS, 161, 394

Farihi, J., Jura, M., \& Zuckerman, B. 2009, ApJ, 694, 805

Girven, J., Gänsicke, B. T., Steeghs, D., \& Koester, D. 2011, MNRAS, 417, 1210

Handler, G. 2001, MNRAS, 323, 43

Hermes, J. J., Charpinet, S., Barclay, T., et al. 2014, ApJ, 789, 85

Kepler, S. O., Castanheira, B. G., Saraiva, M. F. O., et al. 2005, A\&A, 442, 629

Kjeldsen, H. \& Frandsen, S. 1992, PASP, 104, 413

Koester, D. \& Kepler, S. O. 2015, A\&A, 583, 86

Landolt, A. U. 2013, AJ, 146, 131

Liebert, J., Wesemael, F., Hansen, C. J., et al. 1986, ApJ, 309, 241

Limoges, M. M., Bergeron, P., \& Lépine, S. 2015, ApJS, 219, 19

Lindegren, L., Hernández, J., Bombrun, A., et al. 2018, A\&A, 616,2

Marinoni, S., Pancino, E., Altavilla, G., et al. 2016, MNRAS, 462,3616

Mukadam, A. S., Mullally, F., Nather, R. E., et al. 2004, ApJ, 607, 982

Mullally, F., Thompson, S. E., Castanheira, B. G., et al. 2005, ApJ, 625, 966

Paunzen, E. \& Vanmunster, T. 2016, AN, 337, 239

Provencal, J. L., Shipman, H. L., \& MacDonald, J. 2005, ApJ, 627,418

Reegen, P., Gruberbauer, M., Schneider, L., \& Weiss, W. W. 2008, A\&A, 484, 601

Robinson, E. L. \& Winget, D. E. 1983, PASP, 95, 386

Rolland, B., Bergeron, P., \& Fontaine, G. 2018, ApJ, 857, 56

Romero, A. D., Amaral, L. A., Klippel, T., et al. 2019, MNRAS, 490,1803

Romero, A. D., Kepler, S. O., Córsico, A. H., Althaus, L. G., \& Fraga, L. 2014, RMxAC, 44, 54

Salaris, M., Althaus, L. G., \& García-Berro, E. 2013, A\&A, 555,96

Stetson, P. B. 1987, PASP, 99, 191

Tremblay, P. E., Ludwig, H. G., Steffen, M., \& Freytag, B. 2013, A\&A, 559, 104

Voss, B., Koester, D., Napiwotzki, R., Christlieb, N., \& Reimers, D. 2007, A\&A, 470, 1079

Wagner, R. M., Sion, E. M., Liebert, J., Starrfield, S. G., \& Zotov, N. 1986, PASP, 98, 552

Wegner, G. \& Koester, D. 1985, ApJ, 288, 746

Werner, K., Dreizler, S., Heber, U., et al. 1995, A\&A, 293, 75

Werner, K., Rauch, T., \& Kruk, J. W. 2018, A\&A, 609, 107

Wilson, T. G., Farihi, J., Gänsicke, B. T., \& Swan, A. 2019, MNRAS, 487, 133

Winget, D. E., Nather, R. E., Clemens, J. C., et al. 1994, ApJ, 430, 839

Zuckerman, B., Melis, C., Klein, B., Koester, D., \& Jura, M. 2010, ApJ, 722, 725 
Marek Dróżdż: Mt. Suhora Observatory, Pedagogical University, Podchorążych 2, 30-084 Kraków, Poland.

Gerald Handler: Nicolaus Copernicus Astronomical Center, Bartycka 18, 00-716 Warsaw, Poland.

Jan Janík and Ernst Paunzen: Department of Theoretical Physics and Astrophysics, Masaryk University, Kotlăřská 2 , 61137 Brno, Czech Republic (epaunzen@physics.muni.cz).

Sean O’Brien: Space Telescope Science Institute, 3700 San Martin Drive, Baltimore, MD 21218, USA.

Monika Rode-Paunzen: BAS:IS, Austrian Academy of Sciences Dr. Ignaz Seipel-Platz 2, 1010 Vienna, Austria.

Todd K. Watson: Information Technology, Southwestern University, Georgetown, TX, USA.

Zora Zemanová: High School, Velehradská tř́́da 218, 68601 Uherské Hradiště, Czech Republic. 